\title{
FGF2 Induces ERK Phosphorylation Through Grb2 and PKC during Quiescent Myogenic Cell Activation
}

\author{
Yosuke Nagata $^{1,2^{*}}$, Yusuke Honda ${ }^{2}$, and Ryoichi Matsuda ${ }^{2}$ \\ ${ }^{1}$ Komaba Organization for Educational Development, College of Arts and Sciences, The University of Tokyo, \\ 3-8-1 Komaba, Meguro-ku, Tokyo 153-8902, Japan, 2Department of Life Sciences, Graduate School of Arts \\ and Sciences, The University of Tokyo, 3-8-1 Komaba, Meguro-ku, Tokyo 153-8902, Japan
}

\begin{abstract}
Satellite cells are muscle-resident stem cells, which are located beneath the basement membrane of myofibers. Because the number of satellite cells is normally constant, there must be a tight regulation of satellite cell activation and self-renewal. However, the molecular mechanisms involved in satellite cell maintenance are largely unknown, and thus have become the subject of extensive study these days. Although RNA interference with a small interfering RNA has been widely used to investigate the role of specific gene products, inefficient knockdown of Grb2 expression occurred in quiescent reserve cells, a model for quiescent satellite cells, by ordinary transfection protocol. In this study we report that pretreatment with trypsin greatly enhanced siRNA delivery into quiescent reserve cells, resulting in efficient silencing of Grb2 expression. By applying a combination of Grb2-silencing and protein kinase $C$ inhibitors, we demonstrated that extracellular signal-regulated kinase (ERK) phosphorylation induced with fibroblast growth factor 2 (FGF2) was dependent on both Grb2 and protein kinase $\mathrm{C}$ (PKC) with different kinetics. We concluded that the PKC-mediated pathway contributes to rapid initiation and termination of ERK phosphorylation, while the Grb2-mediated pathway contributes to delayed and sustained ERK phosphorylation.
\end{abstract}

Key words: $\quad$ C2C12/satellite cell/Grb2/PKC/siRNA

\section{Introduction}

Satellite cells are muscle-resident stem cells, which are located beneath the basement membrane of myofibers (Mauro, 1961). Adult myofibers are formed by fusion of hundreds of mononucleated myoblasts produced by proliferation of satellite cells. Satellite cells are normally quiescent, but become active in response to certain stimuli, such as injury, overload, and exercise. Activated satellite cells then enter the cell cycle to produce large numbers of myogenic precursor cells, which fuse with existing myofibers

\footnotetext{
*To whom correspondence should be addressed: Yosuke Nagata, Room 25B, Building 101, The University of Tokyo, 3-8-1 Komaba, Meguro-ku, Tokyo 153-8902, Japan.

Tel: +81-3-5465-8820, Fax: +81-3-5465-8821

E-mail: cynagata@mail.ecc.u-tokyo.ac.jp

Abbreviations: BrdU, 5-bromo-2'-deoxyuridine; BSA, bovine serum albumin DMEM, Dulbecco's Modified Eagle Medium; dsRNA, double strand RNA; ERK, extracellular signal-regulated kinase; FBS, fetal bovine serum; FGF2, fibroblast growth factor 2; GAPDH, glyceraldehyde-3-phosphate dehydrogenase; HGF, hepatocyte growth factor; IGF, insulin-like growth factor; PBS, phosphate buffered saline; PI3K, phosphoinositide 3-kinase; PKC, protein kinase C; PLC, phospholipase C; PMA, phorbol 12-myristate 13-acetate; siRNA, small interfering RNA; sMyHC, sarcomeric myosin heavy chain.
}

or each other to form new myofibers (reviewed in Zammit et al., 2006). Because the number of satellite cells is normally constant, there must be tight regulation of satellite cell activation and self-renewal (reviewed in Charge and Rudnicki, 2004). Certain growth factors, such as fibroblast growth factor (FGF), hepatocyte growth factor (HGF), and insulin-like growth factor (IGF) are reported to participate in satellite cell activation (Johnson and Allen, 1995; Tatsumi et al., 1998; Sheehan and Allen, 1999; YablonkaReuveni et al., 1999; Rochat et al., 2004). However, the molecular mechanisms involved in satellite cell activation are largely unknown.

Extracellular signal-regulated kinase (ERK) plays an important role in the control of various cellular responses, including cell proliferation, differentiation, and survival. Ligand binding to growth factor receptors stimulates the receptor tyrosine kinase activity. An adaptor protein Grb2 interacts with activated receptor tyrosine kinases either directly or indirectly via other proteins such as Shc (Margolis, 1999), insulin receptor substrate (Sun et al., 1993) and fibroblast growth factor receptor substrate (Kouhara et al., 1997), and recruits the guanine nucleotide releasing factor Sos. The Grb2/Sos complex facilitates the 
exchange of GDP for GTP on small G-protein Ras at the plasma membrane, resulting in the activation of Raf/ MEK/ ERK pathway (reviewed in Schlessinger, 2000). Although the Grb2/Sos complex plays a central role in ERK activation, other factors, including phosphoinositide 3-kinase (PI3K) /Akt and phosphorlipase C (PLC)/protein kinase C (PKC), could also contribute to the phosphorylation of ERK1/2 via distinct pathways (Grammer and Blenis, 1997; Shu et al., 2002). Because the precise mechanisms of reserve cell activation are largely unknown, understanding what signaling pathways are responsible for the activation of ERK1/2 is of great importance.

These days, RNA interference has been used widely to investigate the function of genes of interest. Successful transfection of cells with a small interfering RNA (siRNA) causes suppression of specific gene expression, thus enabling gene function analysis. siRNA-mediated RNA interference in quiescent myogenic cells would make a significant contribution to the understanding of the precise mechanisms in satellite cell activation. Most studies, however, have attempted siRNA transfection using proliferating cells (Elbashir et al., 2002). In other words, there are few studies using siRNA transfection of quiescent cells. There are several works achieving gene silencing in quiescent cells by transfection of proliferating cells with siRNAs followed by making cells quiescent by serum withdrawal (Asano et al., 2005; Tullai et al., 2007); however, this procedure is not suitable for silencing the genes which are involved in cell proliferation or survival because it will cause significant reduction in the number of quiescent cells. Therefore, an efficient means of siRNA delivery in quiescent cells was required for the purpose. In fact, it was shown that gene silencing did work well in quiescent human bladder carcinoma cells (Nabatiyan and Krude, 2004). However, they also reported that the higher concentrations of siRNA required caused cell death probably by off-target effect. Therefore, we sought a condition for efficient transfection for quiescent myogenic cells with lower concentrations of siRNA. In this study, we demonstrated that pre-treatment with trypsin dramatically improved the efficiency of siRNA delivery and eventually made it possible to silence gene expression in quiescent reserve cells. The efficiency of siRNA delivery was monitored with fluorescent-labeled siRNA, and gene silencing was carried out with siRNAs at $5 \mathrm{nM}$ against Grb2.

In this study, we showed that FGF2 induced DNA synthesis in quiescent reserve cells, a model of satellite cells, in a manner dependent on ERK1/2. Furthermore, we demonstrated that ERK phosphorylation induced with FGF2 was dependent on both the Grb2- and the PKC-mediated pathway with different kinetics by efficient gene silencing of Grb2 in quiescent reserve cells.

\section{Materials and Methods}

\section{Cell culture and drug treatment}

C2C12 myogenic cells (Yaffe and Saxel, 1977; Blau et al., 1983) were maintained in Dulbecco's Modified Eagle Medium (DMEM) (Gibco, Grand Island, NY) containing 20\% fetal bovine serum (FBS) (Equitech-Bio, Kerrville, TX), 25 mM HEPES, $100 \mathrm{U} / \mathrm{ml}$ penicillin, and $100 \mu \mathrm{g} / \mathrm{ml}$ streptomycin (Gibco) at $37^{\circ} \mathrm{C}$ in $5 \%$ $\mathrm{CO}_{2}$. To induce differentiation and produce reserve cells, $5 \times 10^{4}$ cells were seeded on a $35-\mathrm{mm}$ tissue culture dish in growth medium, and $24 \mathrm{~h}$ later, the medium was replaced with serum-free differentiation medium (DMEM supplemented with $10 \mu \mathrm{g} / \mathrm{ml}$ insulin, $5 \mu \mathrm{g} / \mathrm{ml}$ transferrin, $5 \mathrm{ng} / \mathrm{ml}$ sodium selenite, $1 \mathrm{mg} / \mathrm{ml}$ bovine serum albumin (BSA) (Sigma-Aldrich, St Louis, MO), and $25 \mathrm{mM}$ HEPES). To isolate reserve cells from myotubes, $\mathrm{C} 2 \mathrm{C} 12$ cells were cultured in differentiation medium for 4-5 days and then only the myotubes were detached with $0.05 \%$ trypsin (Gibco) in phosphate buffered saline (PBS) containing $\mathrm{CaCl}_{2}$ and $\mathrm{MgCl}_{2}$ $(\mathrm{PBS}(+))$, for $5 \mathrm{~min}$ at $37^{\circ} \mathrm{C}$.

\section{siRNA transfection}

Two sets of pre-designed siRNA duplexes (Stealth siRNA) as well as a negative control duplex were obtained from Invitrogen (Carlsbad, CA). Transfections were performed with Lipofectamine RNAiMAX (Invitrogen) on 35-mm-dishes according to the manufacturer's instructions. siRNA diluted in Opti-MEM and Lipofectamine RNAiMAX diluted in Opti-MEM were mixed and added to cells. The final concentrations of siRNA in the culture media were $5 \mathrm{nM}$. The sequences for Grb2 siRNAs were as follows: 5'-CCGTGTCCAGGAACCAGCAGATATT-3' (\#1) and 5'-CCTGTGGGTGGTGAAGTTTAATTCT-3' (\#2). BLOCK-iT red fluorescent oligo was used instead of these siRNAs to monitor the delivery of siRNA into cell nuclei.

\section{Western blotting}

Cells were fixed with $10 \%$ trichloroacetic acid, and then lysed in SDS-sample buffer (50 mM Tris-HCl, pH 6.8, 2\% SDS, 10\% glycerol, $50 \mathrm{mM}$ dithiothreitol, $0.0025 \%$ bromophenol blue), followed by boiling for $3 \mathrm{~min}$. Ten micrograms of protein were analyzed by $12.5 \%$ SDS-polyacrylamide gel electrophoresis and transferred to polyvinylidene fluoride membranes. Membranes were blocked with Odyssey Blocking Buffer (LI-COR Biosciences, Inc., Lincoln, $\mathrm{NE}$ ) and incubated overnight with primary antibodies. Membranes were washed three times and incubated with Alexa Fluor 680 or IRDye-conjugated secondary antibodies and analyzed with an Odyssey Infrared Imaging System (LI-COR). Primary antibodies used were monoclonal mouse anti-Grb2 (clone 81/GRB2; BD Transduction Laboratories, San Jose, CA), monoclonal mouse anti- $\alpha$-tubulin (clone DM1A, Sigma-Aldrich), polyclonal rabbit anti-ERK1/2 (Promega, Madison, WI), monoclonal mouse antiphospho-ERK1/2 (clone E10, Cell Signaling Technology, Danvers, MA), and polyclonal rabbit anti-phospho-PKC (pan) (Cell Signaling Technology). 


\section{RT-PCR}

Total RNA was isolated with RNAisoPlus reagent (Takara Bio Inc., Shiga, Japan) as specified by manufacturer. One microgram of RNA was used for the reverse transcription reactions using Superscript III First-Strand Synthesis System for RT-PCR (Invitrogen). Aliquots of cDNA were placed in a total volume of $10 \mu \mathrm{l}$ in Platinum Blue PCR SuperMix (Invitrogen). The PCR parameters were $94^{\circ} \mathrm{C}$ for $15 \mathrm{~s}, 55^{\circ} \mathrm{C}$ for $15 \mathrm{~s}$, and $72^{\circ} \mathrm{C}$ for $15 \mathrm{~s}$, for 25 cycles (Grb2) or 30 cycles (Grb2). Five microliter of the PCR products was electrophoresed on $2 \%$ agarose gel containing SYTO60 dye (Invitrogen). The gel was scanned and analyzed with an Odyssey Infrared Imaging System (LI-COR). Primers used in this paper were as follows: glyceraldehyde-3-phosphate dehydrogenase (GAPDH), 5'-AACTTTGGCATTGTGGAGG-3' and 5'-ACACATTGGGGGTAGGAACA-3'; Grb2, 5'-GAGCCAAGGCAGAAGAAATG-3' and 5'-CTTCACCACCCACAGGAAAT-3'.

\section{Immunofluorescent staining}

Cells were fixed with $10 \%$ formalin in PBS for $15 \mathrm{~min}$, and then permeabilized with $0.5 \%$ Triton X-100. Cells were then incubated with mouse anti-sarcomeric myosin heavy chain (sMyHC) antibody (clone MF20). After washes in PBS, primary antibody binding was visualized with Alexa Fluor 488-conjugated secondary antibody (Molecular Probes, Eugene, OR) for $30 \mathrm{~min}$ before washing and mounting in Mowiol mounting medium containing $100 \mathrm{ng} / \mathrm{ml}$ Hoechst 33258.

\section{Bromodeoxyuridine incorporation}

C2C12 cells were fed with fresh DMEM containing $10 \mu \mathrm{M} 5$ bromo-2'-deoxyuridine (BrdU). After incubation for $24 \mathrm{~h}$, cells were fixed with $4 \%$ paraformaldehyde in PBS, followed by permeabilization with $0.5 \%$ Triton X-100. After $2 \mathrm{~N}$ hydrochloric acid treatment for $10 \mathrm{~min}$ at room temperature, cells were immunostained for BrdU with rat anti-BrdU antibody (clone BU1/75, Abcam, Cambridge, MA) as described above. In each experiment, 5 randomly selected fields were photographed and the numbers of total and BrdU-positive nuclei were counted. Each field contained approximately 200 nuclei on the average.

\section{Results}

Quiescent satellite cells play central roles in skeletal muscle regeneration and repair. $\mathrm{C} 2 \mathrm{C} 12$ cells, the most commonly used skeletal muscle cell line, generate quiescent satellite cell-like cells as well as differentiated myotubes (Lindon et al., 1998; Yoshida et al., 1998). Reserve cells are mitotically quiescent but can be activated to enter the cell cycle with FBS, judging from BrdU incorporation (Fig. 1A). Reserve cells would presumably be activated with certain growth factors, such as FGF, HGF, and IGFs, which was reported to participate in satellite cell activation (Johnson
A

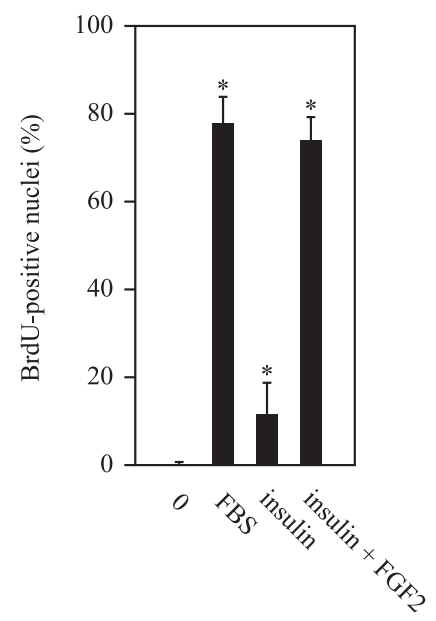

B



Fig. 1. FGF2-induced cell cycle entry of $\mathrm{C} 2 \mathrm{C} 12$ reserve cells. Reserve cells were prepared by culturing $\mathrm{C} 2 \mathrm{C} 12$ cells in serum-free differentiation medium for 5 days, and fed with BSA/DMEM containing $10 \mu \mathrm{M} \mathrm{BrdU}$ in the absence or presence of $10 \% \mathrm{FBS}, 10 \mu \mathrm{g} / \mathrm{ml}$ insulin or $25 \mathrm{ng} / \mathrm{ml} \mathrm{FGF} 2$. Cells were fixed with $10 \%$ formaldehyde after $24 \mathrm{~h}$ in culture, and then subjected to immunodetection of $\mathrm{BrdU}(\mathrm{A})$. After reserve cells were treated with MEK inhibitor U0126 at indicated concentrations, they were stimulated with a combination of $10 \mu \mathrm{g} / \mathrm{ml}$ insulin and $25 \mathrm{ng} / \mathrm{ml} \mathrm{FGF2}$. BrdU incorporation was examined after $24 \mathrm{~h}$ (B). Data presented are the mean percentage of $\mathrm{BrdU}$ positive nuclei $\pm \mathrm{SD}$ from three independent experiments. Asterisks indicate that data are statistically significant using a $t$ test $(\mathrm{P}<0.01)$ compared to unstimulated cells (A) or U0126-untreated cells (B).

and Allen, 1995; Tatsumi et al., 1998; Sheehan and Allen, 1999; Yablonka-Reuveni et al., 1999; Rochat et al., 2004). As shown in Fig. 1A, reserve cells were also activated with FGF2, a well-known regulator of myogenic cell proliferation (Olwin et al., 1994; Sheehan and Allen, 1999; Yablonka-Reuveni et al., 1999), in the presence of insulin (Fig. 1A). FGF2-induced BrdU incorporation in reserve cells was significantly suppressed with U0126, an inhibitor for MEK, suggesting the involvement of ERK $1 / 2$ in reserve cell activation induced with FGF2 (Fig. 1B).

The aim of this study was to define the signaling systems responsible for the initiation and maintenance of ERK1/2 activation induced with FGF2 in reserve cells. Here, we especially focused on the PKC- and Grb2-dependent pathways by using inhibitor and/or siRNA. siRNA-mediated gene silencing was easily and reproducibly carried out in proliferating/differentiation $\mathrm{C} 2 \mathrm{C} 12$ cells. We first tried to transfect proliferating $\mathrm{C} 2 \mathrm{C} 12$ cells with siRNAs and confirmed efficient silencing of the target. Two independent sequences of siRNA duplexes against Grb2 (\#1 and \#2) were tested in this study. After cultured in serum-rich growth medium for $24 \mathrm{~h}, \mathrm{C} 2 \mathrm{C} 12$ cells were transfected with siRNAs in serum-free differentiation medium. At $48 \mathrm{~h}$ after transfection, $\mathrm{C} 2 \mathrm{C} 12$ cells were harvested for detection of 
A

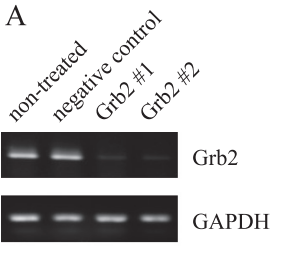

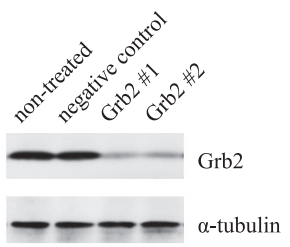
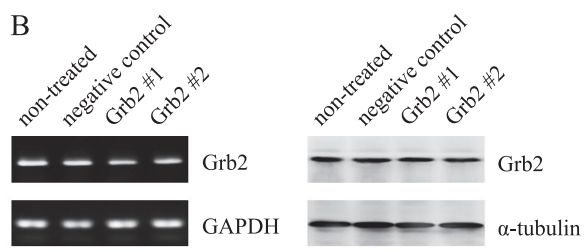

C

Transfected on Day0


Transfected on Day2
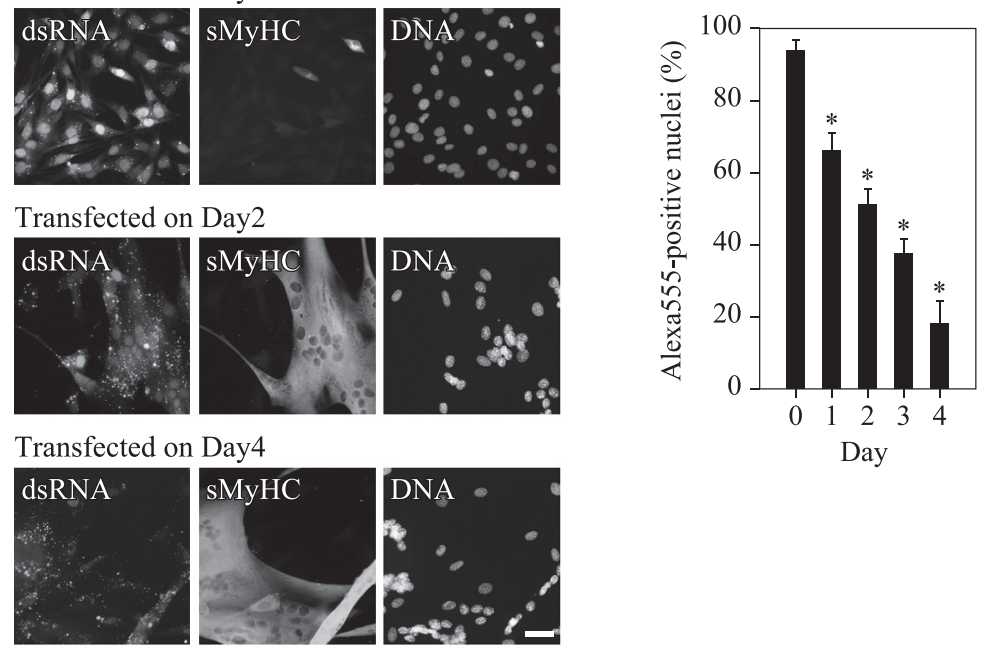

D


pre-treatment with trypsin
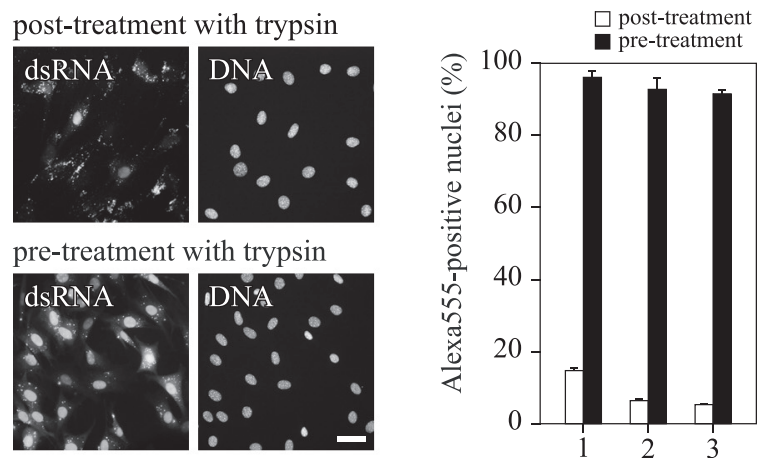

day(s) after transfection

E



Fig. 2. Transfection of Grb2 siRNA into proliferating or quiescent $\mathrm{C} 2 \mathrm{C} 12$ cells. (A and B) Proliferating $\mathrm{C} 2 \mathrm{C} 12$ cells (A) or quiescent $\mathrm{C} 2 \mathrm{C} 12$ cells cultured for 4 days in differentiation medium were transfected with siRNAs, and harvested at 2 days after the transfection for RT-PCR or Western blotting analysis. In contrast to clear decrease in Grb2 expression in proliferating cells, the effect of gene silencing was negligible. siRNAs used in experiment were negative control and 2 independent sequences for Grb2 (\#1 and \#2). GAPDH and $\alpha$-tubulin were used as internal control for RT-PCR and Western blotting, respectively. (C) To monitor the efficiency of siRNA delivery, C2C12 cells were transfected with fluorescent labeled dsRNA at the same time or 1-4 day(s) after serum withdrawal. Cells were fixed at $24 \mathrm{~h}$ after transfection and immunostained for sarcomeric myosin heavy chain (sMyHC) as well as counterstained for DNA. Although transfection on Day0 resulted in efficient dsRNA delivery, the efficiency dropped significantly on Day2 and became much less on Day4. Bar: $50 \mu \mathrm{m}$. The percentage of red fluorescent oligo positive nuclei after the transfection was determined and represented as a graph. Bars indicate SD from three independent experiments. Asterisks indicate that data are statistically significant using a $t$ test $(\mathrm{P}<0.01)$ compared to the transfection on Day0. (D) C2C12 cells cultured in differentiation medium for 4 days were transfected with fluorescent labeled dsRNA. At that time, C2C12 cells formed multinucleated myotubes and mononucleated reserve cells. Mild trypsinization detaches myotubes only, thus leaving reserve cells on culture plate. When trypsin treatment was carried out after the transfection, just before the fixation, only a few cells incorporated siRNA into their nuclei. In contrast, trypsin treatment before transfection greatly enhanced the efficiency of siRNA delivery. Bar: $50 \mu \mathrm{m}$. The percentage of fluorescent dsRNA oligonucleotide-positive cells treated with trypsin after transfection (blank column) and cells treated with trypsin before transfection (filled column) were represented as a graph. Bars indicate SD from three independent experiments. (E) Reserve cells were transfected with siRNAs as described above, harvested 3 days thereafter, and analyzed for Grb2 expression by RT-PCR and Western blotting. As expected, gene silencing of Grb2 was clearly observed at both mRNA- and protein-level. 
Grb2 at mRNA level by RT-PCR as well as at protein level by Western blotting analysis. As shown in Fig. 2A, mRNA expression was markedly reduced with Grb2-specific siRNA. Negative control siRNA, which was designed to minimize sequence homology to any known vertebrate transcript, showed virtually no effect. Furthermore, Western blotting analysis showed Grb2 protein was effectively reduced in Grb2-specific siRNA transfected cells (Fig. 2A).

In order to define the signaling systems responsible for the initiation and maintenance of ERK $1 / 2$ activation induced with FGF2 in reserve cells, we needed to achieve efficient gene silencing in quiescent reserve cells. In contrast to successful siRNA transfection into proliferating cells (Fig. 2A), the transfection of quiescent reserve cells, which were cultured in differentiated serum-free medium for 4 days, resulted in poor silencing of Grb2 expression at both the mRNA- and protein-level (Fig. 2B).

To evaluate the efficiency of siRNA delivery into $\mathrm{C} 2 \mathrm{C} 12$ cells, we used fluorescent-lableled double strand RNA (dsRNA) oligomer. The fluorescent dsRNA oligomer would be incorporated into cell nuclei when the transfection was achieved successfully. We examined on which day $\mathrm{C} 2 \mathrm{C} 12$ cells can be transfected with siRNA after the induction of differentiation. $\mathrm{C} 2 \mathrm{C} 12$ cells were induced to differentiate, and then siRNA transfections were carried out at the same time on 1-4 days thereafter with fluorescent dsRNA oligomer. Cells were fixed at $24 \mathrm{~h}$ after the transfection, and the uptake of fluorescent dsRNA oligomer was assessed. As shown in Fig. 2C, fluorescent signals were readily observed in nuclei when transfection was carried out at the same time of the induction of differentiation. The presence of differentiated myotubes, which expressed sMyHC, became apparent on Day2 or later (Fig. 2C). The efficiency of transfection, as well as fluorescence intensity, was severely reduced in cells cultured in differentiation medium (Fig. 2C). Only $18.1 \%$ of cells incorporated fluorescent dsRNA oligomer when transfection was carried out on Day4 (Fig. 2C). It is worth noting that aggregated fluorescence signals were frequently observed outside cells when siRNA transfection resulted in poor efficiency (Fig. 2C). We also examined how long siRNA remained after $\mathrm{C} 2 \mathrm{C} 12$ cells were transfected with fluorescent dsRNA oligomer on Day0, and found that only $31.4 \%$ of cells possessed fluorescent signal after 5 days (data not shown). Taken together, these results suggest that the ordinary procedure does not work for transfecting quiescent reserve cells with siRNA because of severe reduction in siRNA delivery in these cells.

Mild trypsinization was reported to be useful as to obtain pure reserve cell cultures (Kitzmann et al., 1998). In fact, we have also utilized the technique with some modification and established reproducible isolation (Nagata et al., 2006a). Because conventional transfection did not work for siRNA transfection of reserve cells, we next tried transfection soon after reserve cell isolation. $\mathrm{C} 2 \mathrm{C} 12$ cells were allowed to differentiate for 4 days in differentiation medium. At this time point, approximately $70 \%$ of the cells form multinucleate myotubes, while the rest of the cells remain in an undifferentiated quiescent state (Kitzmann et al., 1998; Yoshida et al., 1998). Cells were treated with $0.05 \%$ trypsin in PBS $(+)$ for $5 \mathrm{~min}$ at $37^{\circ} \mathrm{C}$. Only myotubes were detached from the cell culture plate by this treatment, while reserve cells remain attached to cell dishes. Cells were rinsed with PBS $(+)$ to wash away detached myotubes and remaining trypsin, then fed with DMEM containing siRNA-liposome complexes. Although trypsin-treatment after the transfection did not make any improvement, nuclear-localized fluorescent signals were clearly observed in cells pre-treated with trypsin, and as a consequence, the percentage of fluorescent siRNA-positive cells was maintained at more than $90 \%$ on Day3 after transfection. (Fig. 2D)

Because fluorescent-labeled siRNA were successfully delivered into quiescent reserve cells by trypsin pretreatment, we then tried transfection with siRNAs against Grb2. Reserve cells were harvested for the analysis of the expression of Grb2 at 3 days after transfection. The decrease in mRNA expression was observed at maximum on Day3 (Fig. 2E), and concomitantly, the silencing of gene expression was also observed at protein level (Fig. 2E). Importantly, the efficient silencing of Grb2 expression in quiescent reserve cells was achieved with siRNAs at a concentration of $5 \mathrm{nM}$. There was no obvious cytotoxicity in that condition.

Since we established an efficient siRNA transfection into quiescent reserve cells, we next examined the signaling pathway of reserve cell activation. To examine the involvement of Grb2 and PKC in FGF2-induced reserve cell activation, serum-starved reserve cells were transfected with siRNA against Grb2 as described above, and then after 3 days, cells were stimulated with either FGF2 or phorbol 12myristate 13-acetate (PMA) in the presence or absence of PKC inhibitors. Initially, reserve cells were stimulated with PMA. PMA is a well-known activator of PKC, and thus used to examine the potential role of PKC on ERK1/2 phosphorylation in reserve cells. PMA caused rapid and sustained phosphorylation of ERK1/2 (Fig. 3A), suggesting the presence of a PKC-mediated pathway leading to ERK phosphorylation in reserve cells.

We used a combination of two PKC inhibitors, namely, Gö6976 (Martiny-Baron et al., 1993) and Gö6983 (Gschwendt et al., 1996), to inhibit almost all isoforms of PKC as reported (Shu et al., 2002). After $30 \mathrm{~min}$ pretreatment with PKC inhibitors, reserve cells were stimulated with PMA for $15 \mathrm{~min}$ and then harvested for Western blotting analysis. In reserve cells, the combination of Gö6976 and Gö6983 almost completely suppressed PMAinduced phosphorylation of ERK1/2 (Fig. 3B). Although expression levels of Grb2 were reproducibly decreased to less than $10 \%$ of normal level in Grb2-siRNA treated cells, the silencing of Grb2 did not have any effect on ERK phosphorylation in PMA-stimulated reserve cells (Fig. 3B), 
A

time (min) $\quad \begin{array}{llllll}0 & 2 & 5 & 10 & 20 & 30\end{array}$

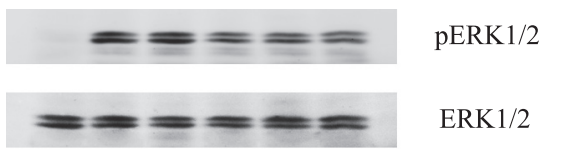

B

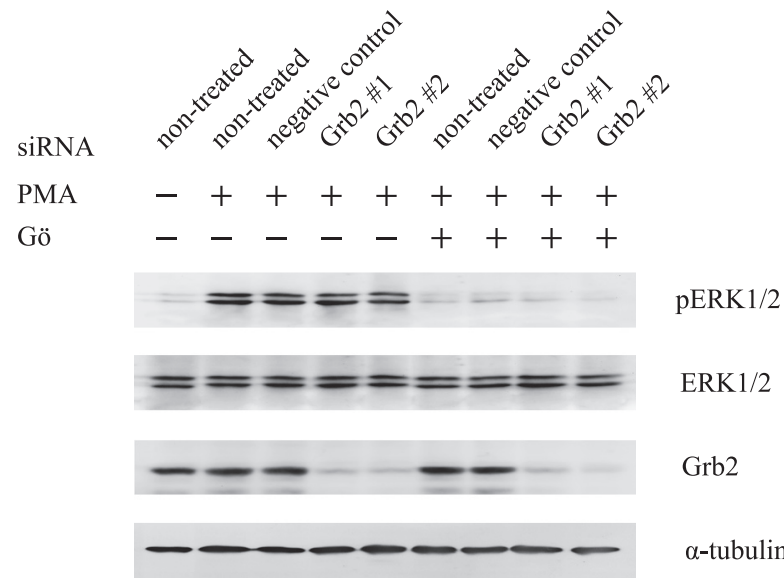

Fig. 3. Analysis of PKC- and Grb2-dependent ERK phosphorylation in PMA-stimulated reserve cells. (A) Serum-starved reserve cells were transfected with siRNA against Grb2 after pretreatment with trypsin, and then 3 days later, cells were fed with 100 nM PMA. After incubation for 2$30 \mathrm{~min}$, cells were harvested for the analysis of ERK phosphorylation. (B) Before PMA was added, reserve cells transfected with siRNAs were treated with a combination of two PKC inhibitors, $1 \mu \mathrm{M}$ Gö6976 and $1 \mu \mathrm{M}$ Gö6983. Fifteen minutes after PMA stimulation, cells were harvested for Western blotting analysis. Expression levels of phospho-ERK1/2, ERK1/2, Grb2, $\alpha$-tubulin were detected with their specific antibodies.

demonstrating that PKC phosphorylated ERK independent of Grb2.

PKC has been reported to mediate peptide growth factor induced signaling pathways (Presta et al., 1989; Nishizuka, 1992; Kim et al., 2003; Clerk et al., 2006).

Indeed, FGF2, as well as PMA, caused phosphorylation of PKC especially with higher molecular weight (Fig. 4A). The PKC antibody used in this study recognizes $\alpha, \beta \mathrm{I}, \beta \mathrm{II}$, $\delta, \varepsilon, \eta$, and $\theta$ isoforms only when phosphorylated at an autophosphorylation site (Keranen et al., 1995). The similar band patterns were reported previously (Sweeney et al., 2001).

FGF2 caused robust and transient phosphorylation of ERK1/2 in reserve cells, detectable within 2 min after the stimulation, peaked around 5 min (Fig. 4B). The suppression of ERK phosphorylation by PKC inhibition was prominent at $2 \mathrm{~min}$. Interestingly, higher levels of phosphorylated ERK1/2 were detected at later time points in Gö-treated cells compared to untreated cells (Fig. 4B). Gene-silencing of Grb2 resulted in decreased levels of ERK phosphoryla- tion at any time point, but certain levels of phosphorylated ERK1/2 were detectable (Fig. 4B).

For close examination of FGF2-induced ERK phosphorylation, cells were treated with the combination of siRNA and PKC inhibitors, and then sampled at 2, 15, and $30 \mathrm{~min}$ after FGF2 stimulation. At 2 min after FGF2 stimulation, phosphorylation of ERK1/2 was significantly suppressed with either siRNA against Grb2 or PKC inhibitors. Almost complete suppression of ERK phosphorylation was observed when cells were transfected with Grb2-specific siRNA followed by treatment with PKC inhibitors (Fig. 4C). Although ERK phosphorylation which was dependent on Grb2 still existed at high levels, sensitivity of PKC inhibition was not observed at $15 \mathrm{~min}$ (Fig. 4C). Only weak signals of phosphorylated ERK1/2 were detectable at 30 min in normal conditions, but PKC-inhibition resulted in higher levels of ERK phosphorylation (Fig. 4C), suggesting the presence of PKC-mediated suppression of receptorcoupled tyrosine kinase activity (Cochet et al., 1984) at later time points. Notably, as shown in Fig. 4C, phosphorylation of ERK1/2 at $30 \mathrm{~min}$ was dependent on Grb2, in contrast to PMA-induced ERK phosphorylation (see Fig. 3B).

Collectively, these results demonstrated that FGF2 induced phosphorylation of ERK1/2 in a manner dependent on both Grb2 adapter protein and PKC with distinct kinetics.

\section{Discussion}

Quiescent satellite cells must be initially activated before participating in skeletal muscle regeneration and repair. Although it is widely known that certain stimuli, such as injury, overload, and exercise, cause activation of satellite cells (reviewed in Charge and Rudnicki, 2004), the molecular mechanisms in the control of satellite cell activation is largely unknown. FGF2 is an established mitogen for myogenic cells and implicated in satellite cell activation (Olwin et al., 1994; Sheehan and Allen, 1999; Yablonka-Reuveni et al., 1999). We showed FGF2 made reserve cells enter the cell cycle partially through the ERK pathway. ERK plays an important role in the control of various cellular responses, including cell proliferation, differentiation, and survival. A variety of growth factors and cytokines are known to activate ERK1/2 via a complicated signaling network. In addition to the Grb2/Sos-mediated pathway, other distinct signaling cascades such as a PLC/PKC- or a PI3K/Akt-mediated pathway could also contribute to ERK activation (reviewed in Schlessinger, 2000).

We showed FGF2 induced phosphorylation of ERK1/2 was mostly dependent on both Grb2 and PKC because ERK phosphorylation was almost, if not completely, suppressed by a combined treatment with Grb2-specific siRNA and PKC inhibitors. Therefore, delayed and sustained phosphorylation of ERK in the presence of PKC inhibitors would reflect mostly the Grb2-mediated pathway. Higher levels of 
A

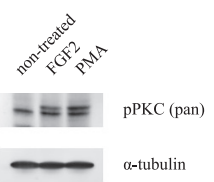

B


$\mathrm{C}$


Fig. 4. Analysis of PKC- and Grb2-dependent ERK phosphorylation in FGF2-stimulated reserve cells. (A) Reserve cells were incubated with either FGF2 for 5 min or PMA for $15 \mathrm{~min}$, and then harvested for the analysis of PKC phosphorylation by Western blotting. The PKC antibody used in this study recognizes $\alpha, \beta I, \beta I I, \delta, \varepsilon, \eta$, and $\theta$ isoforms only when phosphorylated at an autophosphorylation site. (B) FGF2-induced ERK phosphorylation was examined in reserve cells which were in normal condition, treated with PKC inhibitors, or transfected with siRNA against Grb2 (\#1). At 2-30 min after stimulation, cells were harvested for the analysis of ERK phosphorylation. The intensities of phosphor-ERK/ERK were measured and normalized to the value of phosphor-ERK/ERK at $5 \mathrm{~min}$ in FGF2-stimulated reserve cells in normal conditions. Bars indicate SD from three independent experiments. (C) Reserve cells transfected with siRNAs were treated with a combination of PKC inhibitors, and then fed with FGF2. Cells were harvested for Western blotting analysis at 2, 15 or $30 \mathrm{~min}$ after the stimulation. Expression levels of phospho-ERK1/2, ERK1/2, Grb2, and $\alpha$-tubulin were detected with their specific antibodies.

ERK phosphorylation at later time points would demonstrate PKC-mediated suppression of receptor-coupled tyrosine kinase activity (Cochet et al., 1984) in normal conditions. On the other hand, rapid phosphorylation of ERK observed in cells transfected with Grb2 specific siRNAs would mostly demonstrate the PKC-mediated pathway. Taken together, we would conclude that FGF2-caused ERK phosphorylation is dependent on both the Grb2- and the PKC-mediated pathway, and that the PKC-mediated pathway contributes to rapid initiation and termination of ERK phosphorylation, while the Grb2-mediated pathway contributes to delayed and sustained ERK phosphorylation.

In this study, we achieved efficient siRNA transfection of quiescent reserve cells. For siRNA transfection, exponentially growing cells are preferentially used (Elbashir et al., 2002). In fact, we showed that inefficient knockdown of Grb2 expression occurred in quiescent reserve cells by common transfection protocol (Fig. 2B). That is because of poor delivery of siRNA into nuclei as the delivery of fluorescent labeled siRNA was significantly reduced after cells were induced to differentiation by serum withdrawal. Surprisingly though, remarkable improvement of siRNA delivery was observed when transfection was carried out after mild trypsinization. Furthermore, knockdown of Grb2 expression has also occurred efficiently in the trypsin-treated cells before transfection. Several studies have reported siRNA- 
mediated gene silencing in quiescent cells of other cell types by transfection of proliferating cells with siRNAs followed by making cells quiescent by serum withdrawal (Asano et al., 2005; Tullai et al., 2007). However, this protocol was unsuitable for a loss-of-function analysis of Grb2 in reserve cells since the absence of Grb2 in proliferating $\mathrm{C} 2 \mathrm{C} 12$ cells resulted in significant loss of reserve cells (data not shown). Because reserve cells are prepared by serum withdrawal, which causes apoptosis as well as differentiation, it is predicted that the suppression of gene products which control cell proliferation, differentiation, or apoptosis would cause abnormalities in the formation of reserve cells. In contrast, our technique had much less effect on reserve cell formation, thus enabling reliable loss-offunction analyses in reserve cells. It was shown that efficient knockdown was carried out in quiescent human bladder carcinoma cells without extra manipulation except that they used $50 \mathrm{nM}$ of siRNA, five times higher concentrations than normally used, which caused significantly more cell death possibly because of off-target effect (Nabatiyan and Krude, 2004). On the other hand, our method does not require high concentration of siRNA and apparent cytotoxicity was not observed. Because aggregated fluorescent signals were observed only when siRNA transfection resulted in poor efficiency, we conjectured that siRNA/liposome complexes are trapped extracellularly in such conditions. We also speculated trypsin-treatment removed sticky molecules existing in the differentiated cell culture, which thus made siRNA/ liposome complexes accessible to reserve cells. We have previously shown that the expression level of sphingomyelin was decreased as satellite cells were activated (Nagata et al., 2006a), and that sphingosine-1-phosphate, one of sphingomyelin metabolites, contributed to the transition of satellite cells from quiescent to proliferative (Nagata et al., 2006b). Detailed analysis of signaling pathways in the activation of reserve cells, as a model of satellite cells, would become possible by applying siRNA-mediated gene silencing in quiescent reserve cells.

In this study, we revealed that FGF2 caused ERK phosphorylation in a manner dependent on both Grb2 and PKC with different kinetics by efficient gene silencing of Grb2 in quiescent reserve cells. Successful gene silencing in reserve cells would be applicable to other gene products, thus would be useful to examine the molecular mechanisms in the activation of quiescent satellite cells as well as in the maintenance of quiescent state.

Acknowledgments. We would like to thank Dr. P.S. Zammit (King's College London) for critical reading of the manuscript. This research was supported by the MEXT (The Ministry of Education, Culture, Sports, Science and Technology) (Grant-in-Aid for Young Scientists (B), 19770189), Japan. This research was also partially supported by grants $18 \mathrm{~A}-1$ for Nervous and Mental Disorders and H19-kokoro-020 for Research in Brain Science from MHLW (The Ministry of Health, Labour and Welfare), Japan, and grants 17570058 and 14654174 from MEXT, Japan.

\section{References}

Asano, T., Yao, Y., Shin, S., McCubrey, J., Abbruzzese, J.L., and Reddy, S.A. 2005. Insulin receptor substrate is a mediator of phosphoinositide 3-kinase activation in quiescent pancreatic cancer cells. Cancer Res., 65 : 9164-9168.

Blau, H.M., Chiu, C.P., and Webster, C. 1983. Cytoplasmic activation of human nuclear genes in stable heterocaryons. Cell, 32: 1171-1180.

Charge, S.B. and Rudnicki, M.A. 2004. Cellular and Molecular Regulation of Muscle Regeneration. Physiol. Rev., 84: 209-238.

Clerk, A., Aggeli, I.K., Stathopoulou, K., and Sugden, P.H. 2006. Peptide growth factors signal differentially through protein kinase $\mathrm{C}$ to extracellular signal-regulated kinases in neonatal cardiomyocytes. Cell. Signal., 18: 225-235.

Cochet, C., Gill, G.N., Meisenhelder, J., Cooper, J.A., and Hunter, T. 1984. C-kinase phosphorylates the epidermal growth factor receptor and reduces its epidermal growth factor-stimulated tyrosine protein kinase activity. J. Biol. Chem., 259: 2553-2558.

Elbashir, S.M., Harborth, J., Weber, K., and Tuschl, T. 2002. Analysis of gene function in somatic mammalian cells using small interfering RNAs. Methods, 26: 199-213.

Grammer, T.C. and Blenis, J. 1997. Evidence for MEK-independent pathways regulating the prolonged activation of the ERK-MAP kinases. Oncogene, 14: 1635-1642.

Gschwendt, M., Dieterich, S., Rennecke, J., Kittstein, W., Mueller, H.J., and Johannes, F.J. 1996. Inhibition of protein kinase C mu by various inhibitors. Differentiation from protein kinase c isoenzymes. FEBS Lett., 392: 77-80.

Johnson, S.E. and Allen, R.E. 1995. Activation of skeletal muscle satellite cells and the role of fibroblast growth factor receptors. Exp. Cell Res., 219: 449-453.

Keranen, L.M., Dutil, E.M., and Newton, A.C. 1995. Protein kinase C is regulated in vivo by three functionally distinct phosphorylations. Curr. Biol., 5: 1394-1403.

Kim, H.J., Kim, J.H., Bae, S.C., Choi, J.Y., and Ryoo, H.M. 2003. The protein kinase $\mathrm{C}$ pathway plays a central role in the fibroblast growth factor-stimulated expression and transactivation activity of Runx2. J. Biol. Chem., 278: 319-326.

Kitzmann, M., Carnac, G., Vandromme, M., Primig, M., Lamb, N.J., and Fernandez, A. 1998. The muscle regulatory factors MyoD and myf-5 undergo distinct cell cycle-specific expression in muscle cells. J. Cell Biol., 142: 1447-1459.

Kouhara, H., Hadari, Y.R., Spivak-Kroizman, T., Schilling, J., Bar-Sagi, D., Lax, I., and Schlessinger, J. 1997. A lipid-anchored Grb2-binding protein that links FGF-receptor activation to the Ras/MAPK signaling pathway. Cell, 89: 693-702.

Lindon, C., Montarras, D., and Pinset, C. 1998. Cell cycle-regulated expression of the muscle determination factor Myf5 in proliferating myoblasts. J. Cell Biol., 140: 111-118.

Margolis, B. 1999. The PTB Domain: The Name Doesn't Say It All. Trends Endocrinol. Metab., 10: 262-267.

Martiny-Baron, G., Kazanietz, M.G., Mischak, H., Blumberg, P.M., Kochs, G., Hug, H., Marme, D., and Schachtele, C. 1993. Selective inhibition of protein kinase C isozymes by the indolocarbazole Go 6976. J. Biol. Chem., 268: 9194-9197.

Mauro, A. 1961. Satellite cells of skeletal muscle fibres. J. Biophys. Bipchem. Cytol., 9: 493-495.

Nabatiyan, A. and Krude, T. 2004. Silencing of chromatin assembly factor 1 in human cells leads to cell death and loss of chromatin assembly during DNA synthesis. Mol. Cell. Biol., 24: 2853-2862.

Nagata, Y., Kobayashi, H., Umeda, M., Ohta, N., Kawashima, S., Zammit, P.S., and Matsuda, R. 2006a. Sphingomyelin levels in the plasma membrane correlate with the activation state of muscle satellite cells. $J$. Histochem. Cytochem., 54: 375-384. 
Nagata, Y., Partridge, T.A., Matsuda, R., and Zammit, P.S. 2006b. Entry of muscle satellite cells into the cell cycle requires sphingolipid signaling. J. Cell Biol., 174: 245-253.

Nishizuka, Y. 1992. Intracellular signaling by hydrolysis of phospholipids and activation of protein kinase C. Science, 258: 607-614.

Olwin, B.B., Hannon, K., and Kudla, A.J. 1994. Are fibroblast growth factors regulators of myogenesis in vivo? Prog. Growth Factor Res., 5: $145-158$.

Presta, M., Maier, J.A., and Ragnotti, G. 1989. The mitogenic signaling pathway but not the plasminogen activator-inducing pathway of basic fibroblast growth factor is mediated through protein kinase $\mathrm{C}$ in fetal bovine aortic endothelial cells. J. Cell Biol., 109: 1877-1884.

Rochat, A., Fernandez, A., Vandromme, M., Moles, J.P., Bouschet, T., Carnac, G., and Lamb, N.J. 2004. Insulin and wnt1 pathways cooperate to induce reserve cell activation in differentiation and myotube hypertrophy. Mol. Biol. Cell, 15: 4544-4555.

Schlessinger, J. 2000. Cell signaling by receptor tyrosine kinases. Cell, 103: 211-225.

Sheehan, S.M. and Allen, R.E. 1999. Skeletal muscle satellite cell proliferation in response to members of the fibroblast growth factor family and hepatocyte growth factor. J. Cell. Physiol., 181: 499-506.

Shu, X., Wu, W., Mosteller, R.D., and Broek, D. 2002. Sphingosine kinase mediates vascular endothelial growth factor-induced activation of ras and mitogen-activated protein kinases. Mol. Cell. Biol., 22: 7758-7768.

Sun, X.J., Crimmins, D.L., Myers, M.G., Jr., Miralpeix, M., and White, M.F. 1993. Pleiotropic insulin signals are engaged by multisite phosphorylation of IRS-1. Mol. Cell. Biol., 13: 7418-7428.
Sweeney, C., Fambrough, D., Huard, C., Diamonti, A.J., Lander, E.S., Cantley, L.C., and Carraway, K.L., 3rd 2001. Growth factor-specific signaling pathway stimulation and gene expression mediated by ErbB receptors. J. Biol. Chem., 276: 22685-22698.

Tatsumi, R., Anderson, J.E., Nevoret, C.J., Halevy, O., and Allen, R.E. 1998. HGF/SF is present in normal adult skeletal muscle and is capable of activating satellite cells. Dev. Biol., 194: 114-128.

Tullai, J.W., Chen, J., Schaffer, M.E., Kamenetsky, E., Kasif, S., and Cooper, G.M. 2007. Glycogen synthase kinase-3 represses cyclic AMP response element-binding protein (CREB)-targeted immediate early genes in quiescent cells. J. Biol. Chem., 282: 9482-9491.

Yablonka-Reuveni, Z., Seger, R., and Rivera, A.J. 1999. Fibroblast growth factor promotes recruitment of skeletal muscle satellite cells in young and old rats. J. Histochem. Cytochem., 47: 23-42.

Yaffe, D. and Saxel, O. 1977. Serial passaging and differentiation of myogenic cells isolated from dystrophic mouse muscle. Nature, 270: $725-727$

Yoshida, N., Yoshida, S., Koishi, K., Masuda, K., and Nabeshima, Y. 1998. Cell heterogeneity upon myogenic differentiation: down-regulation of MyoD and Myf-5 generates 'reserve cells'. J. Cell Sci., 111: 769-779.

Zammit, P.S., Partridge, T.A., and Yablonka-Reuveni, Z. 2006. The skeletal muscle satellite cell: the stem cell that came in from the cold. $J$. Histochem. Cytochem., 54: 1177-1191.

(Received for publication, November 6, 2009, accepted, April 22, 2010 and published online, May 1, 2010) 